Mastering

Business Law 
Macmillan Master Series

\begin{tabular}{|c|c|}
\hline Accounting & German \\
\hline Advanced English Language & German 2 \\
\hline Arabic & Hairdressing \\
\hline Astronomy & Human Biology \\
\hline Banking & Italian \\
\hline Basic Management & Italian 2 \\
\hline Biology & Japanese \\
\hline British Politics & Manufacturing \\
\hline Business Communication & Marketing \\
\hline Business Law & Mathematics \\
\hline Business Microcomputing & Mathematics for Electrical and Electronic \\
\hline C Programming & Enginearing \\
\hline Catering Science & Modern British History \\
\hline Catering Theory & Modern European History \\
\hline Chemistry & Modern World History \\
\hline COBOL Programming & Pascal Programming \\
\hline Commerce & Philosophy \\
\hline Computer Programming & Photography \\
\hline Computers & Physics \\
\hline Databases & Psychology \\
\hline Economic and Social History & Pure Mathematics \\
\hline Economics & Restaurant Service \\
\hline Electrical Engineering & Science \\
\hline Electronic and Electrical Calculations & Secretarial Procedures \\
\hline Electronics & Social Welfare \\
\hline English as a Foreign Language & Sociology \\
\hline English Grammar & Spanish \\
\hline English Language & Spanish 2 \\
\hline English Literature & Spreadsheets \\
\hline English Spelling & Statistics \\
\hline French & Study Skills \\
\hline French 2 & Word Processing \\
\hline
\end{tabular}




$\int_{\text {Musiness Law }}^{\text {Mastering }}$

Second edition

\section{Terry Price}


@ Terry Price 1982, 1989, 1995

All rights reserved. No reproduction, copy or transmission of this publication may be made without written permission.

No paragraph of this publication may be reproduced, copied or transmitted save with written permission or in accordance with the provisions of the Copyright, Designs and Patents Act 1988, or under the terms of any licence permitting limited copying issued by the Copyright Licensing Agency, 90 Tottenham Court Road, London W1P 9HE.

Any person who does any unauthorised act in relation to this publication may be liable to criminal prosecution and civil claims for damages.

First published 1982 by Pan Books as

Practical Business Law

First published as Mastering Business Law 1989

Reprinted four times

Second edition 1995

Published by

MACMILLAN PRESS LTD

Houndmills, Basingstoke, Hampshire RG21 2XS

and London

Companies and representatives

throughout the world

ISBN 978-0-333-62048-9

ISBN 978-1-349-13549-3 (eBook)

DOI 10.1007/978-1-349-13549-3

A catalogue record for this book is available from the British Library.

$\begin{array}{llllllllll}10 & 9 & 8 & 7 & 6 & 5 & 4 & 3 & 2 & 1\end{array}$

$\begin{array}{rrrrrrrrrr}04 & 03 & 02 & 01 & 00 & 99 & 98 & 97 & 96 & 95\end{array}$ 
Acknowledgements

viii

Introduction

ix

Table of cases

$\mathbf{x}$

Table of statutes (Acts of Parliament)

1 Law and the courts in England and Wales

1.1 Criminal and civil law distinguished

1.2 Criminal and civil courts in England and Wales

2 Sources of the law $\quad 22$

$\begin{array}{ll}2.1 & \text { Legislation } \\ 2.2 & 24\end{array}$

$\begin{array}{lll}2.2 & \text { Precedent } & 28\end{array}$

2.3 European Community law 30

$\begin{array}{lll}2.4 & \text { Custom } & 31\end{array}$

3 European Community law 33

$\begin{array}{lll}3.1 & \text { Treaties } & 34\end{array}$

3.2 Regulations, directives and decisions 36

3.3 Decisions of the Court of Justice of the European

4 Procedure in civil actions and legal personnel 41

4.1 Procedure in civil actions 41

4.2 Personnel of the law 49

5 Business agreements (I) $\quad \mathbf{5 8}$

$5.1 \quad$ Essential requirements in a contract $\quad 58$

5.2 The main contractual elements 65

6 Business agreements (II) 85

6.1 Terms and conditions $\quad 85$

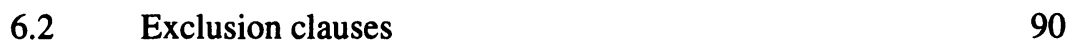

6.3 Discharge of contracts 95

6.4 Remedies for breach of contract 98

7 Employment law 106

$\begin{array}{lll}7.1 & \text { Nature of employment contracts } & 106\end{array}$

7.2 Terms of contracts of employment 114

$\begin{array}{ll}7.3 & \text { Unfair dismissal and redundancy }\end{array}$ 
8 Agency 135

8.1 Contract of agency 136

8.2 Duties of principal and agent 138

8.3 How agency is terminated 139

9 Consumer law 141

9.1 Sale of Goods Act 1979 and Supply of Goods and Services Act 1982

9.2 Trade Descriptions Act 1968 and 1972

9.3 Consumer credit 151

10 Insurance law 163

10.1 Utmost good faith 165

10.2 Insurable interest 166

$\begin{array}{lll}10.3 & \text { Indemnity } & 168\end{array}$

11 Torts (I): negligence $\quad 171$

11.1 The tort of negligence 171

11.2 Possible defences against a negligence action 176

12 Torts (II): trespass, nuisance, defamation and passing off 184

$\begin{array}{lll}12.1 & \text { Trespass } & 184\end{array}$

$\begin{array}{ll}12.2 & \text { Nuisance } \\ 12.3 & 190\end{array}$

12.3 Defamation 192

12.4 Passing off 196

13 Safety at work 198

13.1 Health and Safety at Work Act $1974 \quad 198$

13.2 Civil action available to employees injured at work 201

14 Business organisation (I): sole traders, partnerships and companies 206

14.1 Sole trader and partnership 206

14.2 Formation of a company 213

15 Business organisation (II): privatisation, merger and monopoly control 219

$\begin{array}{ll}15.1 & \text { Public corporations and privatisation } \\ 15.219\end{array}$

15.2 Competition law 221

15.3 Competition and the European Union 223

16 Insolvency law: bankruptcy and liquidation 228

$\begin{array}{lll}16.1 & \text { Bankruptcy } & 228\end{array}$

16.2 Liquidation 233 
17 The law and banking $\quad 237$

$\begin{array}{lll}17.1 & \text { Cheques } & 237\end{array}$

17.2 Negotiable instruments 243

17.3 Protection against forged endorsements 244

18 Business property (I): buying and owning a business 247

$\begin{array}{lll}18.1 & \text { Buying a business } & 247\end{array}$

19 Business property (II): patents, copyright and data protection 258

19.1 Patents, registered designs and trademarks 258

$\begin{array}{lll}19.2 & \text { Copyright } & 262\end{array}$

$\begin{array}{lll}19.3 & \text { Data protection } & 265\end{array}$

$\begin{array}{llr}20 & \text { Review } & 268\end{array}$

$\begin{array}{lr}\text { Answers } & 277\end{array}$

$\begin{array}{lr}\text { Further reading } & 312\end{array}$

$\begin{array}{ll}\text { Index } & 314\end{array}$ 
Acknowledgements are due to the following:

Deborah Nicholson, my daughter, a solicitor who made many constructive suggestions and kept me well informed regarding changes in the law; Rachel Tennant and Julie Price, my daughters, who assisted me in the preparation of the index and various tables; Simon Beckwith, barrister, for some interesting suggestions regarding judicial precedent and insurance law; Dr Angela Conyers, Director of Library Services at Canterbury Christ Church College and her staff for the help given in locating so much information; Norma Featherstone who provided considerable secretarial assistance; and finally to my wife, Sheila, who assisted me greatly in the preparation and whose encouragement made the work so much easier.

Any mistakes or omissions remain of course my sole responsibility.

Terry Price

The author and publishers wish to thank the following who have kindly given permission for the use of copyright material: The Controller of Her Majesty's Stationery Office for extracts from the Sale of Goods Act 1979, Courts Act 1971 and the Unfair Contract Terms Act 1977 and SI 917; the Solicitors' Law Society plc for the Standard Agreement form and other material; and Times Newspapers PLC for 'Women's Entitlement to Equal Pay', The Times, May 1988, 'Estate agent owes duty of Care', The Times, February 1994, 'Battle of the leyland cypresses', The Times, September 1994. 
Mastering Business Law incorporates changes in the law that have been made up to December 1994 and is intended for anyone who wishes to gain an understanding of some of the legal aspects relating to business.

The book will be very useful for anyone studying business courses which include business law, and is of particular relevance to GNVQ/BTEC, NVQ/RSA/LCCI/City and Guilds courses. In addition, the book will be of considerable value to students preparing for GCSE, A Level and professional examinations in business studies and law.

It is important to remember that many changes take place in the law which reflect the changes taking place around us. Readers should try to ensure that they keep up to date. The Times and Guardian publish regular accounts of decided cases and will also provide reliable information on new legislation. 
$\mathbf{A}$

AKZO Chemiw $v$. Commission [1985] 225

Alcock $v$. Chief Constable of South Yorkshire [1991] 15

Anderton $v$. Ryan [1985] 9

Appleson $v$. Littlewoods Ltd [1939] 63

Arterial Caravans Ltd $v$. Yorkshire Insurance Co [1973] 166

Ashbury Railway Carriage Co $v$. Riche [1875] 216

Atlas Express Ltd $v$. Kafco [1989] 82

Attorney General v. Lindi St Claire (Personal Services) [1989] 217

B

Balfour $v$. Balfour [1919] 78

Barber $v$. Guardian Royal Insurance [1990] 119

Barnett $v$. Chelsea and Kensington Hospital Management Committee [1969] 175

Barcabe v. Edwards [1983] 158

Barclays Bank Ltd $v$. W J Sims \& Sons and Cooke Ltd [1980] 240

Bartlett $v$. Sydney Marcus Ltd [1965] 43

Beale $v$. Taylor [1967] 144

Bettini v. Gye [1874-80] $\quad 87$

Billings (A.C.) \& Sons Ltd $v$. Riden [1958] 182

Bollinger J.v. Costa Brava Co [1961] 197

Bostik Itd $v$. Sellotape GB Ltd [1994] 29

Bourhill $v$. Young [1943] $\quad 180$

British Coal v. Smith and Others [1994] 122

British Leyland Plc v. Commission [1987] 226

British Railways Board $v$. Herrington [1972] 15

British Steel Corporation (BSC) v. Cleveland Bridge and Engineering
Co Ltd.

Brown $v$. SE Hampsire Health Authority [1983] 131

Byrne $v$. Van Tienhoven [1880] 68

C

C(a minor) v. director of Public Prosecutions [1994] 29

Capital Finance Co Ltd $v$. Bray [1964] 160

Carlhill v. Carbolic Smoke Ball co [1893] 59

Carlson $v$. Post office [1981] 117

Carrington $v$. Therm-a-Star [1983] 131 
Cassidy $v$. Ministry of Health [1951] 113

Comet Radiovision Services $v$. Farnell Tanberg Ltd [1971] 223

Commission of European Communities v. United Kingdom [1988] 31

Commission of European Communities $v$. United Kingdom [1994] 134

Commission $v$. France (re french nurses) [1984] 34

Commission $v$. Italy (re Housing aid) [1986] 36

Commissioners of Customs and Excise v. Gerhart Schindler and another (1994) 40

Cowan $v$. Le Trésor [1989] 34

Cresswell v. IRC [1984] 116

Curtice v. London City and Midland Bank [1908] 240

D

Dawson $v$. Palter [1984] 260

Dekker $v$. VJV Centrum [1991] 119

Deeny and others $v$. Gooda Walker Ltd and others [1994] 173

D \& C Builders Ltd $v$. Rees [1966]

DF Estates Ltd $v$. The Church Commissioners 175

Donogue $v$. Stevenson [1932] 29

$\mathbf{E}$

Electrolux Ltd $v$. Hudson [1977] 258

Elliott $v$. Saunders [1994] 189

Erewash Borough Council $v$. Ilketon Consumer Co-operative

$\begin{array}{ll}\text { Society Ltd [1988] } & 7\end{array}$

$\mathbf{F}$

Fisher $v$. Bell [1961] 27

Ford $v$. Warwickshire County Council [1983] 125

Freeman \& Lockyer $v$. Buckhurst Park Properties Ltd [1964] 137

$\begin{array}{ll}\text { Froom } v \text {. Butcher } & 179\end{array}$

G

Gardiner $v$. Sevenoaks RDC [1950] 27

Gibson $v$. Manchester City Council [1979] 56

Gran Gelato $v$. Richcliff [1992] 52

R W Green $v$. Cade Bros [1978] 94

$\mathbf{H}$

Halsey $v$. Esso Petroleum Co Ltd [1961] 191

Harmer $v$. Cornelius [1858] 116

Harrison $v$. Kent County Council 1995

Harse v. Pearl Assurance Co Ltd [1904] 167

Haspell $v$. Rostron \& Johnson Ltd [1976] 118

Hayward $v$. Cammell Laird Ltd [1988] 120

Hedley Byrne $v$. Heller \& Partners [1964] 118 
Hertz v. Aldi Marked [1991]

Hill v. Chief Constable of West Yorks Police [1988]

Hivac $v$. Park Royal Scientific Instruments Ltd [1946]

Holden $v$. Bradwell [1985]

Holwell Securities Ltd $v$. Hughes [1971]

Home Office $v$. Dorset Yacht Club [1976]

Howe $v$. Tesco Stores [1974]

Hubbock $v$. Wilkinson [1899]

Hudgell Yeates \& Co $v$. Watson [1978]

\section{I}

IAZ International Belgium NV v. Commission [1983] 225

Inland Revenue Commissioners $v$. Hinchy [1960] 27

International Factors $v$. Rodriguez [1979] 189

Introductions Ltd $v$. National Provincial Bank [1969] 216

$\mathbf{J}$

John Summers \& Sons Ltd $v$. Frost [1955]

204

Jones and Others $v$. Wright [1991]

$\mathbf{K}$

Krell $v$. Henry [1903] 96

Ketley $v$. Scott [1981] 158

$\mathbf{L}$

Lewis v. Surrey County Council [1987] $\quad 125$

Liverpool City Council $v$. Irwin [1977] $\quad 89$

London Borough of Bromley $v$. Greater London Council [1982]

London Joint Stock Bank v. Macmillan [1918]

M

Mahesan $v$. Malaysian Government Housing Society [1974] 139

Marshall $v$. Southampton Area Health Authority [1986] 119

Mercantile Credit Co Ltd $v$. Garrod [1962] 210

Macarthys Ltd $v$. Smith [1981] $\quad 30$

McCullagh $v$. Lane Fox and Partners [1994] 43

Midland Bank Trust Co Ltd $v$. Hett, Stubbs \& Kemp [1979] 52

Morrish $v$. Henlys [Folkestone] Ltd [1973] 115

Morris $v$. Murray [1991] $\quad 15$

$\mathbf{N}$

Nash $v$. Inman [1908]

Nemes v. Allen [1977] $\quad 107$

Northam $v$. Barnet London Borough Council [1978] 28

North Yorkshire County Council v. Ratcliffe and Others [1994] 122 
0

O. Brien $v$. Associated Fire Alarms Ltd [1969] 129

Olley v. Marlborough Court Ltd [1949] 91

O’Kelly v. Trust House Forte [1983] 114

$\mathbf{P}$

Parkes Classic Confectionery v. Ashcroft [1973] 111

$\begin{array}{ll}\text { Pearce } v \text {. Brooks [1866] } & 79\end{array}$

Pearce $v$. Secretary of State for Defence [1988] 15

$\begin{array}{lr}\text { Pepper } v \text {. Webb [1969] } & 115\end{array}$

Perry (Howard) \& Co Ltd $v$. British Railways Board [1980] 189

Pharmaceutical Society of Great Britain v. Boots Cash Chernists [1953] 56

Photo Production Ltd $v$. Securicor Transport Ltd [1980] 94

$\begin{array}{lr}\text { Property Guards Ltd } v \text {. Taylor [1982] } & 108\end{array}$

Poussard $v$. Spiers \& Pond [1876] 87

$\begin{array}{lr}\text { Price } v \text {. the Civili Service Commission [1987] } & 119\end{array}$

Publishers' Association v. Commission [1992] 225

Pyke $v$. Hamilton Bank [1950] 193

$\mathbf{R}$

R v. Allen [1871] 27

R v. Brentwood Justices ex Parte Nicholls [1990] 5

R v. Criminal Injuries Compensation Board ex Parte Thompstone [1993] 19

R v. Ford [1989] 56

R v. Marylebone Justices ex Parte Yasmin Farrog [1980] 7

R v. Mason [1981] $\quad 56$

$\mathrm{R} v$. Lawrence [1981] $\quad 8$

R $v$. Sutcliffe [1981] 5

R v. Pigg [1983] 56

R v. McIlkenny [1991] $\quad 8$

R v. Shivpuri [1986] 9

Reading v. Attorney General [1951] 117

Ready Mix Concrete Ltd $v$. Ministry of Pension 114

and National Insurance [1968]

Rentokil $v$. Mackin [1989] 131

$\begin{array}{lr}\text { Richley } v \text {. Faull [1965] } & 174\end{array}$

Rogers $v$. Parish (Scarborough) [1987] 25

Rondel $v$. Worsley [1969] $\quad 52$

Rose $v$. Plenty [1976] 183

Ross $v$. Caunter [1980] $\quad 52$

Rowland $v$. Divall [1923] 142

$\mathbf{S}$

Saloman v. Saloman \& Co Ltd [1897] 215

Sayers $v$. Harlow UDC [1958] $\quad 178$

Saif Ali v. Sydney Mitchell \& Co [1980] 52

Shields $v$. Coomes [1979] 107 
xiv Table of cases

Shine $v$. General Guarantee Corporation Ltd [1988] 143

Stanton $v$. Jones $1984 \quad 186$

Stevenson \& Sons $v$. AG furCartoggen Industrie [1918] 211

Stevenson, Jordan and Harrison Ltd v. Macdonald Evans [1952] 117

$\begin{array}{ll}\text { Smith } v \text {. Eric Bush [1989] } & 172\end{array}$

Spicer Ltd $v$. Mansell [1970] 209

Steer $v$. Durable Rubber Co Ltd [1958] 181

$\begin{array}{ll}\text { Stekel } v \text {. Ellice [1973] } & 210\end{array}$

Stennet $v$. Hancock [1939] 182

Stevenson \& Sons $v$. AG für Cartoggen Industrie [1918] 211

Stevenson, Jordan and Harrison Ltd $v$. Macdonald and Evans [1952] 263

Stoke on Trent Council v. B \& Q plc [1993] 17

$\mathbf{T}$

$\begin{array}{ll}\text { Targett } v \text {. Torfaen BC [1991] } & 175\end{array}$

Taylor $v$. Alidair [1978] 131

Taylor v. Kent County Council [1969] 129

Tayside Regional council $v$. McIntosh [1982] 116

Thameside Health Authority $v$. Hyde [1981] 15

$\begin{array}{ll}\text { Torr } v \text {. British Railways Board [1977] } & 108\end{array}$

$\mathbf{V}$

Victoria Laundry [Windsor] Ltd v. Newman Industries Ltd [1949] 99

$\mathbf{W}$

Warner Brothers Pictures Incorporated $v$. Nelson [1973] 101

Watteau $v$. Fenwick [1893] 202 Wylie v. Dee \& Co [1978] 136

Warninck $v$. Townend [1979] 196

Waugh $v$. Clifford \& Sons [1982] 137

Wilhelm $v$. BundesKartellamt [1969] 224

Williams v. Roffey Bros and Nicholls (Contractors) [1990] 71

$\begin{array}{lr}\text { Wills } v \text {. Bowley [1982] } & 27\end{array}$

Wilson $v$. Pickering [1946] 243

Wings Ltd $v$. Ellis $\quad 149$

$\begin{array}{ll}\text { Winsor } v \text {. Schroeder [1979] } & 211\end{array}$

Wylie $v$. Dee \& Co (Menswear) Ltd [1978] 119 


\section{Table of statutes (Acts of Parliament)}

B

Bankruptcy Act 1986

221

Bill of Exchange Act 1882

237

Business Names Act 1985

207

C

Cheques Act 1957 and 1992

Children Act 1989

Companies Acts 1981 \& 1985

Computer Misuse Act 1990

Consumer Credit Act 1974

Consumer Protection Act 1987

181

Contracts [Applicable Law] Act 1990

103

Contracts of Employment Acts 1963 \& 1972

Copyright Act 1982 \& 1983

258

Copyright, Designs and Patents Act 1988

258

Copyright (Computer Software) Amendment Act 1985

264

County Courts Act 1984

Courts Act 1971

Courts and Legal Services Act $1990 \quad 12$

Criminal Justice Act 1987

Crown Proceedings Act 1947

D

Data Protection Act 1984

Deed of Arrangement Act 1914

Defective Premises Act 1972

$\mathbf{E}$

Employers' Liability (Defective Equipment) Act 1969

Employment Acts 1980, 1982, 1988, 1989, 1990 and 1994

Employment Protection (Consolidation) Act 1978

Environmental Protection Act 1990

Equal Pay Act 1970

European Communities Act 1992 
xvi Table of statutes (Acts of Parliament)

F

Factories Act 1961

Fair Trading Act 1973

Finance Act 1960

H

Health and Safety at Work Act 1974

I

Income Tax Act 1952

Insolvency Acts 1985 and 1986

J

Juries Act 1974

Juries (Disqualification) Act 1984

$\mathbf{L}$

Landlord and Tenant Act 1954

256

Latent Damage Act 1986

Law of Property [Miscellaneous Provisions] Act 1989

Law Reform (Frustrated Contracts) Act 1954

Law Reform Miscellaneous Provisions Act 1970

Life Assurance Act 1774

Limitation Act [1980]

Limited Partnership Act 1907

Local Government Act 1972

M

Mental Health Act

Merchant Shipping Act 1970

Minors Contract Act 1987

Monopolies and Merges Act 1973

$\mathbf{0}$

Occupier's Liability Act 1957 and 1984

Offences against the Person Act 1861

$\mathbf{P}$

Partnership Act 1890

Patent Act 1977

Pharmacy and Poisons Act 1949

Police and Criminal Evidence Act 1984

Post Office Act 1969

$\mathbf{R}$

Race Relations Act 1976 
Resale Price Maintenance Act $1964 \quad 223$

Resale Prices Act $\quad 223$

$\begin{array}{ll}\text { Redundancy Payments Act } 1965 & 126\end{array}$

Registered Designs Act $1949 \quad 260$

Registration of Business Names Act $1916 \quad 208$

Rehabilitation of Offenders Act 1974

Restrictive Trade Practices Act $1976 \quad 80$

Restriction of Offensive Weapons Act 1961226

$\begin{array}{ll}\text { Road Traffic Act } 1972 & 73\end{array}$

$\mathbf{S}$

Sale of Goods Act 1979

$\begin{array}{lr}\text { Sex Discrimination Act 1975-86 } & 107\end{array}$

Shops Act $1950 \quad 7$

Social Security Act 1975-80

Supply of Goods (Implied Terms) Act 1979

Supply of Goods and Services Act 1982

$\mathbf{T}$

Town Police Clauses Act $1847 \quad 27$

$\begin{array}{ll}\text { Trade Description Act } 1968 \text { \& } 1972 & 147\end{array}$

Trade Marks [Amendment Act] $1984 \quad 261$

$\begin{array}{ll}\text { Trade Union and Labour Relations Act } 1974 & 77\end{array}$

Trade Union Reform and Employment Rights Act 1992

Torts [Interference with Goods] Act $1977 \quad 189$

U

Unfair (Contract Terms Act) 197792 